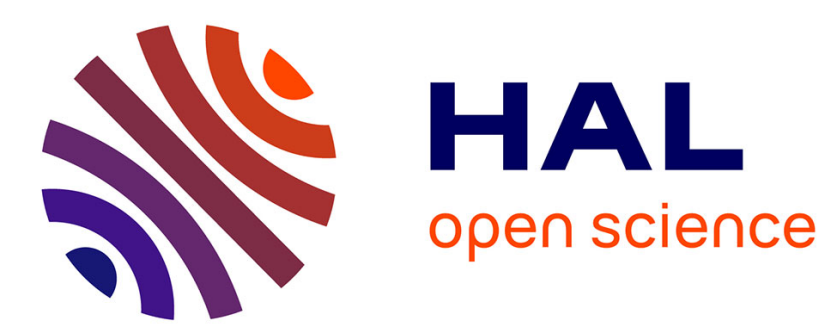

\title{
Numerical Approach for the Assessment of Pressure Generated by Elastic Compression Bandage
}

Fanette Chassagne, Jérome Molimard, Reynald Convert, Pascal Giraux, Pierre D Badel

\section{- To cite this version:}

Fanette Chassagne, Jérome Molimard, Reynald Convert, Pascal Giraux, Pierre D Badel. Numerical Approach for the Assessment of Pressure Generated by Elastic Compression Bandage. Annals of Biomedical Engineering, 2016, 44, pp.3096 - 3108. 10.1007/s10439-016-1597-3 . hal-01380275

\section{HAL Id: hal-01380275 \\ https://hal.science/hal-01380275}

Submitted on 12 Oct 2016

HAL is a multi-disciplinary open access archive for the deposit and dissemination of scientific research documents, whether they are published or not. The documents may come from teaching and research institutions in France or abroad, or from public or private research centers.
L'archive ouverte pluridisciplinaire HAL, est destinée au dépôt et à la diffusion de documents scientifiques de niveau recherche, publiés ou non, émanant des établissements d'enseignement et de recherche français ou étrangers, des laboratoires publics ou privés. 
1 Numerical approach for the assessment of pressure generated by elastic 2 compression bandage

3

4 Fanette Chassagne ${ }^{a, b, c^{*}}$, Jérome Molimard ${ }^{a, b}$, Reynald Convert ${ }^{c}$, Pascal 5 Giraux $^{d}$, Pierre Badel ${ }^{a, b}$

6

${ }^{a}$ Inserm, U1059, Saint-Etienne, F-42023, France

7

${ }^{b}$ Mines Saint-Etienne, Saint-Etienne, F-42023, France

8

${ }^{c}$ Thuasne, BP243, 92307 Levallois-Perret cedex, France

$9{ }^{d}$ Department of Physical Medicine and Rehabilitation, Faculty of Medicine, University 10 Jean Monnet, Saint-Etienne, France

$11{ }^{*}$ Corresponding author. Email : fanette.chassagne@emse.fr 12 
14 Compression of the lower leg by bandages is a common treatment for the advanced

15 stages of some venous or lymphatic pathologies. The outcomes of this treatment 16 directly result from the pressure generated onto the limb. Various bandage

17 configurations are proposed by manufacturers: the study of these configurations

18 requires the development of reliable methods to predict pressure distribution applied

19 by compression bandages. Currently, clinicians and manufacturers have no dedicated

20 tools to predict bandage pressure generation.

21 A numerical simulation approach is presented in this work, which includes patient-

22 specific leg geometry and bandage. This model provides the complete pressure

23 distribution over the leg. The results were compared to experimental pressure

24 measurements and pressure values computed with Laplace's law.

25 Using an appropriate surrogate model, this study demonstrated that such simulation is 26 appropriate to account for phenomena which are neglected in Laplace's law, like

27 geometry changes due to bandage application.

28

29 Keywords: Numerical simulation; leg compression; bandage; subject-specific 


\section{Introduction}

Compression of the lower leg is a common treatment for venous or lymphatic diseases ${ }^{7}$ and can be performed either with elastic stockings or bandages ${ }^{5}$. Bandages are mainly used in the initial steps of the treatment of advanced stages of these pathologies such as legs ulcers or lymphedema ${ }^{2}$. The treatment success depends on the pressure level which is applied on the leg in the supine and standing positions and while walking ${ }^{15,19}$. Even though the efficacy of this treatment, performed either with stockings or bandages, is admitted ${ }^{3,5}$, its action mechanism and the pressure it applies on the leg remain poorly understood, especially in the case of bandages ${ }^{17}$.

The pressure applied on a leg by compression bandage mainly depends on the following parameters:

- bandage components (crepes, padding layer, ...),

- mechanical properties of the different bandage components, such as elastic modulus,

- bandage stretch,

- application technique (spiral, figure-of-eight) and number of layers (overlap),

- leg morphology (local curvature of the limb),

- other parameters (soft tissue mechanical properties, friction between bandage layers, ...).

Different configurations are proposed to clinicians: application of "inelastic" or "elastic" bandages, 2 or 3 layers bandage application technique, combination of 
different types of bandages... The relevance and understanding of these various methodologies needs to be addressed.

For this, research on the mechanical action of elastic compression has been addressed using both experimental and modeling approaches, within the aim of understanding pressure generation and its effects on the leg soft tissues. An effort has been made to measure interface pressure applied by compression stockings or bandages ${ }^{3,8,13,22}$, though in vivo pressure measurements only provide localized information about the applied pressure. However, for now, local measurement methods are significantly more accurate than pressure mapping systems ${ }^{4}$, hence the lack of a convenient method to measure the complete pressure distribution over the leg.

A rough estimation of local pressure field can be obtained with Laplace's law (Equation 2) as a function of the local radius of curvature of the limb, the bandage tension and the number of layers. However this equation does not consider every aspect involved in the mechanism of pressure generation ${ }^{15}$, such as shape changes due to bandage application or position variations. Despite many attempts to improve this law ${ }^{1,25}$, it remains insufficient to quantify the complete pressure distribution ${ }^{24}$.

Numerical simulations were introduced to achieve this goal. However, most of them sought to better understand the action mechanism of compression stockings which behave in a different way than bandage. Among these studies, a first $2 \mathrm{D}$ model was proposed considering a plastic leg, whose mechanical properties were known and whose geometry was obtained from a CT-scan ${ }^{27}$. Experimental pressure measurements were performed on the leg in order to be compared with the results given by the model. In this simulation strategy, the compression stocking was first inflated and then relaxed around the leg. The results they obtained had similar trends 
with experimental results. Later, 3D models were introduced in order to consider the heterogeneity and patient-specificity of the leg soft tissues ${ }^{10}$. The leg geometry was obtained from CT-scan images and the material parameters were identified by an inverse approach based on CT-scans of the patients wearing compression stockings. The main limitation of this simulation was that it did not model the stocking, the pressure applied on the leg being calculated using Laplace's law. Another more recent model was built in order to better understand how the pressure is transmitted through soft tissues and affects veins inside the leg ${ }^{23}$. Its $2 \mathrm{D}$ geometry was obtained from MRI images to separate muscle compartments, main veins and adipose tissue.

To the authors' knowledge, only one attempt to simulate the action of compression bandage was proposed by Al Khaburi et al. ${ }^{1}$. The bandage was not modeled and the pressure exerted on the leg was computed using an improved Laplace's Law.

In short, to explain the action mechanism of compression bandage via the pressure distribution they generate, Laplace's law is not sufficient ${ }^{24}$. On the other hand, local pressure measurements are not sufficient to understand the global action of compression bandages and there is, for now, no reliable technique for pressure mapping over the leg. As a consequence, clinicians and manufacturers currently have no reliable methods to predict pressure distribution applied by compression bandages, hence the need to develop new tools.

This study presents a first subject-specific numerical simulation to model the pressure distribution generated by compression bandages application. This simulation will lead to a more global approach of interface pressure generated by bandages, including 3D local curvature of the leg and its changes with bandage application, and soft tissue 
mechanical properties. The aim of the study was to validate the feasibility and reliability of a finite element (FE) simulation of lower-limb bandage in a simple case, in order to use it later with different bandage configurations.

The approach was based on experimental leg shape and pressure measurements on 5 female subjects for model generation and validation. For each subject, leg geometry was obtained with a 3D optical scanner and was then used to model the leg and the corresponding bandage. Bandage mechanical properties were measured experimentally. The results given by the simulation were then compared to the experimental pressure values. All results were compared to Laplace's Law, as it is, for now, considered as the reference method for interface pressure computation.

\section{Methods}

\section{a. Experimental pressure measurements}

Pressure measurements were performed on the medial and lateral sides of five female subjects' right legs with no known leg-related diseases, following informed consent (see Table 1).

Interface pressure was measured with pneumatic sensors Picopress ${ }^{\circledR}$ (Microlab Elettronica, Nicolò PD, Italy), whose diameter was $50 \mathrm{~mm}$ and discrimination threshold and resolution were $1 \mathrm{mmHg}$. Linearity and hysteresis were tested in a preliminary study and provided excellent results: linearity $R^{2}>0.9999$ and hysteresis $<1.0 \%{ }^{6}$. However, the presence of this sensor modifies the real interface pressure (a measurement always modifies the measurand). A correction method was proposed 
and used to correct the interface pressure values measured in this study (see section below).

Four sensors gave local values of the pressure applied on the leg. They were placed at two different heights on the medial and lateral sides of the leg: two were located at the height of measurement point B1 (lower end of the calf) and two at the height of measurement point $C$ (largest circumference of the calf) on both sides of the leg (Figure $1-(a)$ ). Measurement point B1 was chosen following the recommendations of a consensus paper ${ }^{19}$ while measurement point $C$ was chosen because it is the fleshiest part of the calf. Then the bandage was applied and finally the foot was slightly raised in order to prevent any contact between the examination bed and the calf. After waiting for one minute in this position, pressure values were recorded.

All bandages were applied by the same trained operator in order to limit the discrepancy due to inter-operator variability: a bandager seems constant and repeatable in applying bandages ${ }^{21,14}$.

For each subject, four different bandages were applied on the leg in a spiral pattern (Figure $1-(b)$ and $(c)$ )

- Biflex 16 (B16), a 100 mm wide bandage manufactured by Thuasne, with 2 and 3 layers (respectively $50 \%$ and $66 \%$ overlap),

- Biflex 17 (B17), another 100 mm wide bandage manufactured by Thuasne, with 2 and 3 layers (respectively 50\% and 66\% overlap).

Both have the same textile structure but B17 has a higher elastic modulus than B16 (Table 3).

The manufacturer recommends applying these bandages with a 1.3 stretch (stretch $=L / L_{0}$, with $L$ the applied bandage length and $L_{0}$ its initial length). To guarantee 
that this stretch is reached, a calibration marker was printed on these bandages every $10 \mathrm{~cm}$ by the manufacturer (Figure $1-(\mathrm{d})$ ). It consists in a rectangle which turns into a square when stretch is 1.3 .

In this study, it was important to accurately measure the actual post-application bandage stretch because bandage application may be variable ${ }^{18}$. This was performed by a manual measurement of the distance between each printed marker:

$$
\text { Stretch }=\frac{\text { distance between } 2 \text { markers after application }}{\text { distance between } 2 \text { markers before application }(10 \mathrm{~cm})}
$$

Prior to bandage application and actual stretch measurement, and within the aim of performing the subsequent simulation, the subject's leg geometry was scanned in standing position, with a structured light 3D scanner (Artec ${ }^{\mathrm{TM}} \mathrm{MHT}$ 3D scanner, $\operatorname{Artec}^{\mathrm{TM}}$ Group), whose resolution is $0.5 \mathrm{~mm}$. The experimental pressure measurement is to be compared with the pressure extracted from the simulation; the former corresponds to the average of the pressure over an area corresponding to the sensor surface, and the latter has to be averaged over the same area. In order to accurately define the corresponding area, the sensor location was marked with a sticker that can be distinguished on the 3D scan of the leg without significantly modifying the leg geometry (Figure $3-(d)$ ).

\section{b. Correction of the pressure measurement error due to the sensor}

\section{volume}

Although the pressure sensor is very thin, it can locally modify the curvature of the limb when inflated with the $2.0 \mathrm{~mL}$ air volume required. This curvature modification can then lead to a variation of the pressure applied by the textile. 
In order to properly compare the experimental results with the simulation results (in which the sensor is not modeled), the local pressure variation due to the sensor was numerically investigated. For that, a 2D numerical model was built consisting of a disc representing the leg cross-section, whose radius varied from 30 to $70 \mathrm{~mm}$, on which lied the sensor. The disc and the sensor were modeled as neo-hookean hyperelastic materials. The disc mechanical properties were the same as the living soft tissue mechanical properties used later in the simulation whereas the sensor mechanical properties were chosen in order to have very low shear stiffness and a high volumetric compression modulus (respectively $c_{10}=4.0 \mathrm{kPa}, \mathrm{D}_{1}=0.14 \mathrm{MPa}^{-1}$ and $c_{10}=$ $\left.4.0 * 10^{-3} \mathrm{kPa}, \mathrm{D}_{1}=0.14 * 10^{-3} \mathrm{MPa}^{-1}\right)$. The pressure was applied by a circular textile (modeled with linear beam elements) with a 1.3 stretch, whose stiffness varied from $0.46 \mathrm{MPa}$ to $1.32 \mathrm{MPa}$ (i.e. the equivalent range of elastic moduli used in this study (Table 3)). The friction coefficient between the disc and the sensor was $0.4^{12}$. The textile was inflated, then relaxed thus applying pressure on the disc and the sensor. The measured pressure was computed as the mean pressure on all nodes of the sensor. The reference pressure was the pressure at the opposite side of the disc, where there was no sensor (Figure $2-(a)$ ). The relative error was defined as follows:

$$
\text { Relative error }[\%]=\frac{\text { Measured pressure }- \text { Reference pressure }}{\text { Reference pressure }} * 100
$$

Thanks to this model, it was possible to quantify the measurement error due to the curvature modification (Figure $2-(b)$ ). A $3^{\text {rd }}$ order polynomial correction profile was fitted on these results $\left(R^{2}=0.93\right)$. It can be noticed that the higher the pressure, the lower the error. Indeed, as pressure increases, penetration of the sensor into soft tissues increases, so the curvature modification and the error decrease. All the 
experimental pressure values presented in this study were corrected according to this correction profile.

\section{c. Numerical simulation}

A subject-specific simulation of bandage application was built, whose input data were the subject's leg geometry, the bandage mechanical properties and the application technique.

\section{Leg geometry}

The leg geometry was scanned with a structured light 3D scanner (Artec ${ }^{\mathrm{TM}}$ MHT 3D scanner, Artec ${ }^{T M}$ Group) before bandage application (Figure $\left.3-(a)\right)$. The scan provided a cloud of points used to further reconstruct the external leg geometry model.

The bone holes geometry was based on anatomical slices from the 'Visible Human Server' (Computer Science Department, Peripheral Systems Lab., Ecole Polytechnique Fédérale de Lausanne) (Figure $3-(a)$ ). This bone geometry was scaled to fit the leg geometry and implanted in the leg volume using Boolean operations. These geometrical modeling operations were performed using the software SolidWorks ${ }^{\circledR}$. It was verified in a preliminary study that little variation in the geometry of the bones had negligible influence on the pressure applied onto the skin. Bones being considered as non-deformable, the outer surface of soft tissues in contact with bones was assigned pinned boundary conditions (this mimics the presence of non-deformable material).

The complete leg was then meshed in Abaqus ${ }^{\circledR}$ with 4-node linear tetrahedron hybrid elements (around 600,000 elements, depending on subjects). Previously, a mesh 
convergence study was performed in order to determine the optimal element size of both parts: bandage and leg. Last, leg soft tissues were considered as a single homogeneous material whose constitutive equation and parameters were taken from the literature (Table 2) ${ }^{10}$. A preliminary study confirmed that soft tissue mechanical properties had very little influence on pressure distribution in this simulation (mean variation: $0.20 \pm 3.33 \%$, for $\left.\mathrm{c}_{10}=1-35 \mathrm{kPa}\right)$, justifying this choice.

\section{Bandage geometry}

The only known requirement for modeling the bandage is the 1.3 longitudinal stretch, once it is applied on the leg.

The bandage model geometry, which preserves this 1.3-stretch condition, was built from the leg geometry obtained from the 3D scanner data (Figure 3-(e)).

The shape of the bandage which is applied in a spiral pattern with respectively $50 \%$ or $66 \%$ overlap between successive layers consists in a helix. A custom meshing code $\left(M_{a t l a b}{ }^{\circledR}\right)$ generated the FE mesh of any bandage knowing its width, the number of layers, the leg geometry, and ensuring a bandage stretch equal to 1.3 once the bandage is applied on the scanned leg geometry. The bandage was meshed with 4node shell elements with reduced integration, whose size was determined by the mesh convergence study (about 55,000 elements, depending on subjects and number of bandage layers).

Bandage constitutive equation was considered as orthotropic linear elastic (Equation 1). Its parameters were characterized following the same methodology as described by Demanget ${ }^{9}$, combining different mechanical tests: 
- several pure and plane strain tensile tests, to determine the in-plane orthotropic elastic behavior of the fabrics and, thus, obtain the longitudinal and transverse elastic moduli $\left(K_{1}\right.$ and $\left.K_{2}\right)$, the in-plane Poisson's ratio $\left(v_{21}\right)$, and the in-plane shear modulus G (see Equation 1);

- "nail tests", to determine the out-of-plane bending behavior of the fabrics and, thus, estimate the longitudinal and transverse bending stiffnesses of the fabrics $\left(F_{1}\right.$ and $\left.F_{2}\right)$. All these parameters are linked by the following equation:

Equation 1

$$
\left[\begin{array}{l}
\varepsilon_{11} \\
\varepsilon_{22} \\
\gamma_{12}
\end{array}\right]=\left[\begin{array}{ccc}
\frac{1}{K_{1}} & -\frac{v_{12}}{K_{1}} & 0 \\
-\frac{v_{21}}{K_{2}} & \frac{1}{K_{2}} & 0 \\
0 & 0 & \frac{1}{G}
\end{array}\right]\left[\begin{array}{l}
\sigma_{11} \\
\sigma_{22} \\
\sigma_{12}
\end{array}\right] \quad\left[\begin{array}{l}
M_{11} \\
M_{22} \\
M_{12}
\end{array}\right]=\left[\begin{array}{ccc}
F_{1} & \sim 0 & 0 \\
\sim 0 & F_{2} & 0 \\
0 & 0 & \tau_{12}
\end{array}\right]\left[\begin{array}{l}
\kappa_{11} \\
\kappa_{22} \\
\kappa_{12}
\end{array}\right]
$$

With $\varepsilon_{11}, \varepsilon_{22}$ and $\sigma_{11}, \sigma_{22}$ the strains and the section forces in the in-plane directions, $\gamma_{12}$ and $\sigma_{12}$ the shear strains and section forces, $M_{11}, M_{22}$ and $M_{12}$ the section moments and $\kappa_{11}, \kappa_{22}, \kappa_{12}$ the bending strains.

\section{Simulation strategy}

The simulation of bandage application was performed in 2 steps (Figure 4):

- Bandage dilatation, by imposing a radial displacement (Figure 4-(a));

- Activation of leg to bandage contact followed by bandage relaxation. First, only the radial displacement was relaxed (Figure $4-(b)$ ), then all displacement degrees of freedom were relaxed except both ends of the bandage which were pinned (Figure $4-(c)$ ). 
The contact between the leg and the bandage allowed no penetration and had a friction coefficient equal to $0.4^{12}$. There was no contact between the different bandage layers.

\section{d. Laplace's Law}

Laplace's law (Equation 2) is considered as the reference method for the computation of pressure applied by bandages. Hence, the experimental and simulated pressure values were compared with those obtained using this law:

$$
P=\frac{n T}{r}, \quad T>0, \quad r>0 \quad \text { (Equation 2) }
$$

with $\mathrm{P}$ the pressure $\left(N . \mathrm{mm}^{-2}\right), \mathrm{n}$ the number of layers of the bandage, $\mathrm{T}$ the bandage tension (ratio between the force needed to stretch the bandage and the bandage width) $\left(N . \mathrm{mm}^{-1}\right)$ and $\mathrm{r}$ the local radius of curvature $(\mathrm{mm})$.

To do so, transverse cross sections of the leg external surface were approximated by Fourier polynomials:

$r(\theta, z)=a(z)+\sum_{k=1}^{n}\left[b_{k}(z) \cos (k \theta)+c_{k}(z) \sin (k \theta)\right]$

where $r(\theta, z)$ is the local radius (in the transverse plane) for a given angle $\theta$ and a given height $z$ (cylindrical coordinate system) and $n$ is the degree of the polynomials $(n=5)$.

The radius of curvature was then computed using the following equation ${ }^{10}$ :

$$
R_{C}(\theta, z)=\frac{\left(r^{2}(\theta, z)+r^{\prime 2}(\theta, z)\right)^{\frac{3}{2}}}{r^{2}(\theta, z)+2 r^{\prime 2}(\theta, z)-r(\theta, z) r^{\prime \prime}(\theta, z)}
$$

with $r^{\prime}$ and $r^{\prime \prime}$ the first and second derivatives of the local radius of curvature. 
The pressure given by Laplace's law is theoretically a function of the bandage tension in the transverse plane, $T_{1}$, and that in sagittal and coronal planes, $T_{2}$. Nevertheless, as the radius of curvature in the coronal/sagittal planes, $R_{2}$, is much larger than that in the transverse plane, $R_{1}$, the longitudinal component of Laplace's law was neglected:

$P=\frac{T_{1}}{R_{1}}+\frac{T_{2}}{R_{2}} \quad$ and $R_{2} \gg \gg R_{1}$ and $T_{1} \gg \gg T_{2} \Rightarrow \frac{T_{2}}{R_{2}} \ll \ll \frac{T_{1}}{R_{1}} \quad$ (Equation 5)

The pressure was computed from the leg geometry obtained from the scanner (nondeformed state) and the tension was computed knowing bandage stiffness, number of layers and bandage stretch.

\section{e. Comparison of the different results}

In order to compare the results obtained from the experiments, the simulation and Laplace's law, an attempt was made to fit a linear model on the three data sets. The linear model was inspired from Laplace's Law: $\log (P)=\log (n)+\log (T)+\log (C)$, with the local leg curvature, $C=\frac{1}{r}$. This linear model was modified with the aim of evaluating the linear effects $a_{i}$ and the combined effects $a_{i j}$ of each variable, which may explain the differences between the results sets:

$$
\begin{aligned}
& \log (P)=a_{0}+a_{1} \log (n)+a_{2} \log (T)+a_{3} \log (C)+a_{12} \log (n) \log (T)+ \\
& a_{13} \log (n) \log (C)+a_{23} \log (T) \log (C) \quad \text { (Equation 6) }
\end{aligned}
$$

If the three data sets (given by the experiments, the simulation and Laplace's Law) were identical, then the identified coefficients $a_{i}$ and $a_{i j}$ should be equal for the different sets.

For the simulation, the local curvature $(C)$ was that obtained at the end of the simulation (i.e. in the deformed state) whereas for the experience and Laplace's law the curvature was that in the non-deformed state (as the final geometry is unknown). 
The tension $(T)$ is a function of the bandage stretch. For the data computed with Laplace's Law, the stretch was 1.3. For the experiments and the simulations, the tension was computed with regards to the actual known stretch values. The three variables (tension, curvature and number of layers) were centered and scaled between -1 and 1 before fitting.

\section{f. Statistical analysis}

All the plotted histograms represent the mean value and the $95 \%$ confidence interval. The linear correlation between two samples was evaluated using the coefficient of determination $\mathrm{R}^{2}$, which is equal to the square of the Pearson correlation coefficient between the data (experimental or numerical) and the values given by the linear model. The significance of this coefficient $R^{2}$ can be tested thanks to the variable $t=\sqrt{R^{2} * \frac{n-2}{1-R^{2}}}$, with $n$ the size of the sample. For a zero correlation, this variable $t$ is approximately distributed as a Student's distribution with $n-2$ degrees of freedom. The differences between pressures applied by the different bandages (B16 - 2 layers, B16 - 3 layers, B17 - 2 layers, B17 - 3 layers) were tested with the Kruskal-Wallis oneway analysis of variance and the individual effects were tested with a Mann-Whitney $U$ test $\left(\alpha=\frac{0.05}{\text { number of tests }}\right)$. A non-parametric test for paired samples (Wilcoxon signedrank test) was used to evaluate the differences between:

- Stretches for the two bandages and around the two measurement points,

- Pressures applied by 2-layer and 3-layer bandages and pressures applied by bandages B16 and B17 (for the experience and the simulation).

\section{Results}


The results given by the experiments and the simulation are respectively presented in Figure 5 and Figure 6. They were compared with the pressures computed using Laplace's law, as it is considered as the reference method for interface pressure computation.

The pressure given by Laplace's Law was obviously proportional to the bandage elastic modulus and the number of layers. Therefore the ratio between the pressure applied by the B17 and that applied by the B16 was equal to the ratio of bandage stiffnesses (Figure $5-(c)$ and Figure $6-(b)$ ). Similarly, the pressure applied by a 3-layer bandage was 1.5 times as high as that applied by a 2-layer bandage (Figure $5-(\mathrm{d})$ and Figure 6 (c)). Eventually, the pressure at measurement point B1 was about $15 \%$ as high as that at point $C$ (Figure $5-(e)$ and Figure $6-(d))$.

In the experiments, the measured mean stretch for all bandage applications was equal to $1.30 \pm 0.02$ (Figure 5 - (a)). However, stretches for the B16 and the B17 bandages differed $(p<0.05)$, with respective values of $1.32 \pm 0.03$ and $1.28 \pm 0.03$. In addition, the measured stretch was found to be different between the two measurement points $(p<0.01)$, with higher values at point $C$ (mid-calf) than at point B1 (lower end of the calf) (respectively $1.34 \pm 0.02$ and $1.25 \pm 0.02$ )

The pressure measurement error induced by the presence of the sensor was corrected for all the experimental results presented below. From a general point of view, it can be noticed that pressure increased with the number of layers of the bandage (Figure 5 - (b) and (d)) and with bandage elastic modulus (Figure 5 - (b) and (c)), as expected. Comparing the two types of bandages, the ratio between the pressure exerted by the B17 and that exerted by the B16 was about 1.5 at equal number of layers and with the 
hypothesis that stretches were equal (Figure $5-(c)$ ), whereas the ratio of their stiffnesses is 1.91 (Table 3). Eventually, the applied pressure varied along the leg: the pressure at measurement point $C$ was slightly lower than at point B1 (Figure $5-(e)$ ).

Twenty different simulations were built: 5 female subjects and 4 bandages for each subject. Among these 20 models, 19 simulations reached convergence. An illustration of representative pressure maps is provided in Figure $4-(d)$ and (e). The pressure values which were extracted for comparison to the experimental data consist in the mean pressure over the areas corresponding to the sensors' locations (Figure $3-(d)$ ). These results are presented in Figure 6. Finale bandage stretch was $1.282 \pm 0,008$ when the target stretch was 1.3 (on the undeformed leg), which shows the validity of the bandage geometry used in the simulation and the applied boundary conditions. The pressure increased with the number of layers $(p<0.01)$ and the bandage elastic modulus $(p<0.01)$ (Figure $6-(a)$, (b) and (c)). The pressure applied by the B17 (with the highest elastic modulus) was about 1.8 times as high as the pressure applied by the B16 (Figure $6-(b))$, at equal number of layers and with the hypothesis that bandages stretches were equal. However, the pressure applied by a bandage with a $66 \%$ overlap ( 3 layers) was about 1.4 times the pressure applied by a bandage with a $50 \%$ overlap ( 2 layers) (Figure $6-(c))$. Eventually, the pressure increase between the measurement points $C$ and B1 was about $11 \%$ (Figure $6-(d)$ ).

\section{Discussion}

This numerical approach proposed a new patient-specific simulation of compression bandage application, which is, to the authors' knowledge, the first one to model the bandage. This simulation provides the complete pressure distribution over the leg, 
with regards to the patient's leg morphology, the bandage mechanical properties and the application technique. The results given by the simulation need to be compared with the experiments.

In the experiments, bandage stretch was lower for the B16 than for the B17, which is due to the fact that the B17 is stiffer than the B16 (Table 3), thus harder to stretch. The experiments confirmed that the applied pressure increased with bandage elastic modulus. However, the pressure applied by the B17 was about 1.5 times as high as the pressure applied by the B16, while the B17 is 1.9 times as stiff as the B16 (hence the expected pressure ratio would be around 1.9). For this ratio, it was hypothesized that bandages' stretches were equal, which was not the case in the experiments (Figure 5 - (a)). Nevertheless, this difference in measured stretch does not explain such a large difference between the observed trend and the expected pressure value.

The ratio between the pressure exerted by 2-layer and 3-layer bandages was slightly higher than the expected ratio (1.5), even if the bandage stretch was about the same for both application techniques: $1.29 \pm 0.03$ for the 2-layer application technique and $1.30 \pm 0.03$ for the 3-layer application technique. Even though these pressure measurements were performed on only 5 female subjects, all these trends were in accordance with the results of a previous study ${ }^{6}$, which was performed on 30 subjects (15 men - 15 women) and whose protocol was very close to that previously described. In short, the 3 data sets obtained from the experience, the simulation and Laplace's law showed different trends, especially regarding the influence bandage stiffness or number of layers. A simple way to compare the 3 data sets was to compare the pressure values at measurement point B1 on the medial side of the leg for all subjects ( 
1 Figure 7). Although the differences between the data sets were almost never

2 significant, the trends vary within the different bandages, hence the need to have an

3 overall approach for the comparison of the different results.

4 For this a linear model was fitted on the different data sets. The linear effects $a_{i}$ and

5 the combined effects $a_{i j}$ (Equation 6) were identified for the experiments, the

6 simulation and Laplace's law (Figure 8).

7 The coefficient $a_{0}$ was not discussed because it consists in a constant value due to the 8 conversion between pressure units.

9 As a first observation, these results showed that the effects of the different factors differ within the three data sets (Figure 8). Note, also, that all non-zero effects were 11 significant $(p<0.05)$. The linear effects of the number of layers and the curvature, respectively $a_{1}$ and $a_{3}$, followed the same trends as the slopes showed in the previous results (Figure 5 - (d) and Figure 6 - (c) for the number of layers and Figure $5-$ (c) and

14 Figure 6 - (b) for the curvature). The number of layers had lower impact in the simulation than in the two other data sets. The curvature had larger influence on the pressure computed with Laplace's law than in the simulation and in the experience.

17 This can be explained, at least in part, by the fact that Laplace's law does not consider 18 the curvature modification due the soft tissue compression caused by bandage application.

20 Nevertheless, the linear effect of the bandage tension $a_{2}$ does not follow the same 21 trends as the slopes previously presented (Figure $5-(\mathrm{c})$ and Figure 6 - (b)). Indeed, 22 these slopes represent the ratio between the pressure applied by two bandages with 23 different elastic moduli, applied with the same number of layers and with the 24 hypothesis that their stretches were equal. However, the linear model did not consider 
25 the bandage elastic moduli but the bandage tension, which is a function of the

26 bandage elastic moduli and stretch (Tension $=(1-$ stretch $) *$ elastic modulus $)$.

27 The stretch was constant in the simulation but varied in the experiments (Figure 5 -

28 (a)). Therefore, this stretch variation can explain the difference between the linear 29 effect of the bandage tension (Figure 8) and the trends previously observed in Figure 5 $30-(c)$ and Figure $6-(b)$.

31 More especially, as expected, no combined effect was found in Laplace's law. 32 However, the combined effects of the curvature with the tension and the number of 33 layers, $a_{23}$ and $a_{13}$, play a role in the simulation and the experiments. This could be explained by the curvature modification due to bandage application, which is larger when the number of layers and the tension increase. The bandage applied on the leg tends to make the leg geometry rounder (radii of curvature are evened out).

37 Eventually, in the light of the coefficient of determination $\mathrm{R}^{2}$ characterizing the correlation between the experiments and the linear model $\left(R^{2}=0.8514\right)$, the $\operatorname{limb}$ curvature, the bandage tension and the number of layers are not the only parameters which impact on the interface pressure. Hence, Laplace's law cannot completely explain the pressure distribution applied on a leg by compression bandages.

Limitations

All pressure measurements were performed in a short time after bandage application, so they do not reflect bandage long-term or dynamic effects (over one-day or after walking). 
The curvature modification due to the sensor was only numerically assessed. Several studies have investigated the performance of the pneumatic sensor Picopress, but their conclusions differ ${ }^{20} 26$. However, one of them confirmed that the low pressure

52 values tend to be overestimated when the radius of curvature is too small ${ }^{26}$.

53

54 The interaction between the different bandage layers may play an important role during bandage application. However, it was not taken into account in the simulation.

56

57

An interesting perspective would be to add bandage to bandage interactions in future simulations. In this regard, the simulation of the real wrapping process, i.e. new boundary conditions, would likely be more suitable to account for these interactions.

Soft tissue mechanical properties used in the simulation were taken from the literature ${ }^{10}$ and were identical for the 5 female subjects. As a preliminary study showed, the impact of these properties on the generated pressure is limited. Nevertheless, their effect on pressure transmission through soft tissues is of higher importance, for instance for vein closure ${ }^{11}$. For this reason, it would be relevant to use subject-specific soft tissue mechanical properties.

\section{Conclusion}

Under the more general background of studying different possible bandage configurations, the aim of this study was to propose a patient-specific finite-element model of bandage application to assess pressure distribution applied by elastic compression bandages. A major improvement over Laplace's law was that this simulation models leg shape changes due to bandage application, whereas Laplace's 
72 law only considers the non-deformed state of the leg. Moreover, Laplace's law does

73 not consider any interactions between the bandage and the leg, expect normal

74 pressure.

75 This study evaluated the feasibility and reliability of a numerical simulation and 76 showed that improving the simulation complexity, by taking bandage-to-bandage

77 friction into account, for example, is promising.

78 This patient-specific simulation could then be used to assess pressure distribution over

79 a patient's leg in order to adapt his treatment, by choosing a specific bandage or 80 application technique, and to prevent localized overpressures, which could improve 81 the compliance with the treatment.

\section{Conflict of interest}

83 Thuasne is a medical devices manufacturer. 


\section{References}

1. Al Khaburi, J., A. A. Dehghani-Sanij, E. A. Nelson, and J. Hutchinson. Effect of bandage thickness on interface pressure applied by compression bandages. Med. Eng. Phys. 34:378-385, 2012.

2. Allaert, F.-A. Différentes indications de la compression élastique. Actual. Pharm. 54:14-20, 2015.

3. Amsler, F., T. Willenberg, and W. Blättler. In search of optimal compression therapy for venous leg ulcers: A meta-analysis of studies comparing divers bandages with specifically designed stockings. J. Vasc. Surg. 50:668-674, 2009.

4. Bonnaire, R., M. Verhaeghe, J. Molimard, P. Calmels, and R. Convert. Characterization of a pressure measuring system for the evaluation of medical devices. Proc. Inst. Mech. Eng. [H] 228:1264-1274, 2014.

5. Brizzio, E., F. Amsler, B. Lun, and W. Blättler. Comparison of low-strength compression stockings with bandages for the treatment of recalcitrant venous ulcers. J. Vasc. Surg. 51:410-416, 2010.

6. Chassagne, F., F. Martin, P. Badel, R. Convert, P. Giraux, and J. Molimard. Experimental Investigation of Pressure Applied on the Lower Leg by Elastic Compression Bandage. Ann. Biomed. Eng. 43:2967-2977, 2015.

7. Coleridge-Smith, P. D. Leg ulcer treatment. J. Vasc. Surg. 49:804-808, 2009.

8. Danielsen, L., S. Munk Madsen, L. Henriksen, J. Sindrup, and L. J. Petersen. Subbandage pressure measurements comparing a long-stretch with a short-stretch compression bandage. Acta Derm Venerol 78:201-204, 1998.

9. Demanget, N., S. Avril, P. Badel, L. Orgéas, C. Geindreau, J.-N. Albertini, and J.-P. Favre. Computational comparison of the bending behavior of aortic stent-grafts. J. Mech. Behav. Biomed. Mater. 5:272-282, 2012.

10. Dubuis, L., S. Avril, J. Debayle, and P. Badel. Identification of the material parameters of soft tissues in the compressed leg. Comput. Methods Biomech. Biomed. Engin. 15:3-11, 2012.

11. Frauziols, F., J. Molimard, L. Navarro, P. Badel, M. Viallon, R. Testa, and S. Avril. Prediction of the biomechanical effects of compression therapy by finite element modeling and ultrasound elastography. IEEE Trans. Biomed. Eng. 62:1011-1019, 2015.

12. Gerhardt, L.-C., A. Lenz, N. D. Spencer, T. Münzer, and S. Derler. Skin-textile friction and skin elasticity in young and aged persons. Skin Res. Technol. 15:288-298, 2009.

13. Hafner, J., I. Botonakis, and G. Burg. A comparison of multilayer bandage systems during rest, exercise, and over 2 days of wear time. Arch. Dermatol. 136:857-863, 2000.

14. Logan, R. A., S. Thomas, and E. F. Harding. A comparison of sub bandage pressures produced by experienced and inexperienced bandagers. J. Wound Care 23-26, 1992.

15. Melhuish, J. M., M. Clark, R. Williams, and K. G. Harding. The physics of subbandage pressure measurement. J. Wound Care 9:308-310, 2000. 
16. Milic, D. J., S. S. Zivic, D. C. Bogdanovic, M. M. Jovanovic, R. J. Jankovic, Z. D. Milosevic, D. M. Stamenkovic, and M. S. Trenkic. The influence of different subbandage pressure values on venous leg ulcers healing when treated with compression therapy. J. Vasc. Surg. 51:655-661, 2010.

17. Partsch, H. Evidence based compression therapy. VASA 62 (S/63):1-39, 2003.

18. Partsch, $H$. The use of pressure change on standing as a surrogate measure of the stiffness of a compression bandage. Eur. J. Vasc. Endovasc. Surg. 30:415-421, 2005.

19. Partsch, H., M. Clark, S. Bassez, J.-P. Benigni, F. Becker, V. Blazek, J. Caprini, A. Cornu-Thénard, J. Hafner, M. Flour, M. Jünger, C. Moffatt, and M. Neumann. Measurement of lower leg compression in vivo: recommendations for the performance of measurements of interface pressure and stiffness: consensus statement. Dermatol. Surg. 32:224-232; discussion 233, 2006.

20. Partsch, H., and G. Mosti. Comparison of three portable instruments to measure compression pressure. Int. Angiol. 29:426-430, 2010.

21. Raj, T. B., M. Goddard, and G. S. Makin. How long do compression bandages maintain their pressure during ambulatory treatment of varicose veins? Br. J. Surg. 67:122-124, 1980.

22. Rimaud, D., R. Convert, and P. Calmels. In vivo measurement of compression bandage interface pressures: The first study. Ann. Phys. Rehabil. Med. 57:394-408, 2014.

23. Rohan, C. .-Y., P. Badel, B. Lun, D. Rastel, and S. Avril. Biomechanical response of varicose veins to elastic compression: A numerical study. J. Biomech. 46:599-603, 2013.

24. Schuren, J., and K. Mohr. The efficacy of Laplace's equation in calculating bandage pressure in venous leg ulcers. WOUNDS UK 4:38-47, 2008.

25. Thomas, S. The use of the Laplace equation in the calculation of sub-bandage pressure. Eur. Wound Manag. Assoc. 21-23, 2003.

26. Thomas, S. Practical limitations of two devices used for the measurement of subbandage pressure: Implications for clinical practice. J. Wound Care 23:300-313, 2014.

27. Uhl, J.-F., S. Drapier, I. Gaied, and B. Lun. Pression théorique et pression mesurée in-situ des BMC : apport de l'imagerie médicale et des techniques de simulations numériques. Phlébologie 58:131-138, 2005. 
List of tables

Table 1: Principal characteristics of the 5 female patients

\begin{tabular}{|c|c|c|}
\cline { 2 - 3 } \multicolumn{1}{c|}{} & Mean value & $\begin{array}{c}\text { Standard } \\
\text { deviation }\end{array}$ \\
\hline Age & 38.6 y.o. & 7.8 y.o. \\
\hline $\begin{array}{c}\text { Circumference } \\
\text { at point B1 }\end{array}$ & $32.4 \mathrm{~cm}$ & $2.2 \mathrm{~cm}$ \\
\hline $\begin{array}{c}\text { Circumference } \\
\text { at point C }\end{array}$ & $36.9 \mathrm{~cm}$ & $1.9 \mathrm{~cm}$ \\
\hline
\end{tabular}

Table 2: Soft tissue mechanical properties ${ }^{10}$

\begin{tabular}{|l|ll|l}
\hline Leg soft tissue & $c_{10}=4 \mathrm{kPa}$ & $D_{1}=0.14 M P a^{-1}$ & $U=c_{10}\left(\bar{I}_{1}-3\right)+\frac{1}{D_{1}}\left(J^{e l}-1\right)^{2}$ \\
\hline
\end{tabular}

Table 3 : Bandage mechanical properties

\begin{tabular}{|c|c|c|c|}
\cline { 2 - 4 } \multicolumn{1}{c|}{} & $\begin{array}{c}\text { Longitudinal and } \\
\text { transverse elastic } \\
\text { moduli }\end{array}$ & $\begin{array}{c}\text { Poisson ratio and in- } \\
\text { plan shear modulus }\end{array}$ & $\begin{array}{c}\text { Longitudinal and } \\
\text { transverse bending } \\
\text { stiffnesses }\end{array}$ \\
\hline Biflex ${ }^{\oplus} 16$ & $\mathrm{~K}_{1}=0.232 \mathrm{~N} \cdot \mathrm{mm}^{-1}$ & $\mathrm{~V}_{12}=0.072$ & $F_{1}=0.13 \mathrm{MPa}$ \\
& $\mathrm{K}_{2}=0.417 \mathrm{~N} \cdot \mathrm{mm}^{-1}$ & $\mathrm{G}=0.11 \mathrm{~N} \cdot \mathrm{mm}^{-1}$ & $F_{2}=0.15 \mathrm{MPa}$ \\
\hline Biflex 17 & $\mathrm{~K}_{1}=0.444 \mathrm{~N} \cdot \mathrm{mm}^{-1}$ & $\mathrm{~V}_{12}=0.065$ & $F_{1}=0.41 \mathrm{MPa}$ \\
& $\mathrm{K}_{2}=0.706 \mathrm{~N} \cdot \mathrm{mm}^{-1}$ & $\mathrm{G}=0.21 \mathrm{~N} \cdot \mathrm{mm}^{-1}$ & $F_{2}=0.25 \mathrm{MPa}$ \\
\hline
\end{tabular}


Figure 1 : Location of measurement points (a) - Bandage applied with a $50 \%$ overlap (2 layers) (b) and a 66\% overlap (3 layers) (c) - Bandage calibration marker (rectangle which turns into a square when the stretch is equal to 1.3) (d)

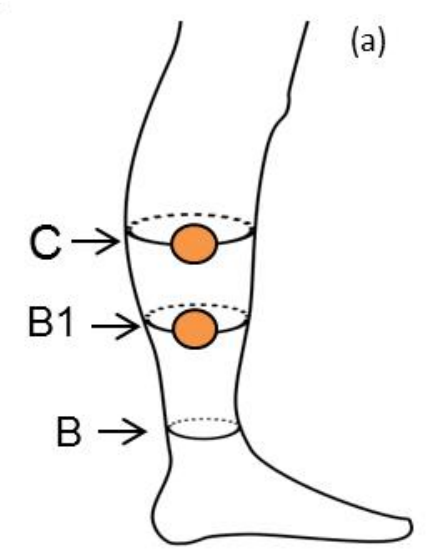

(b)

(c)
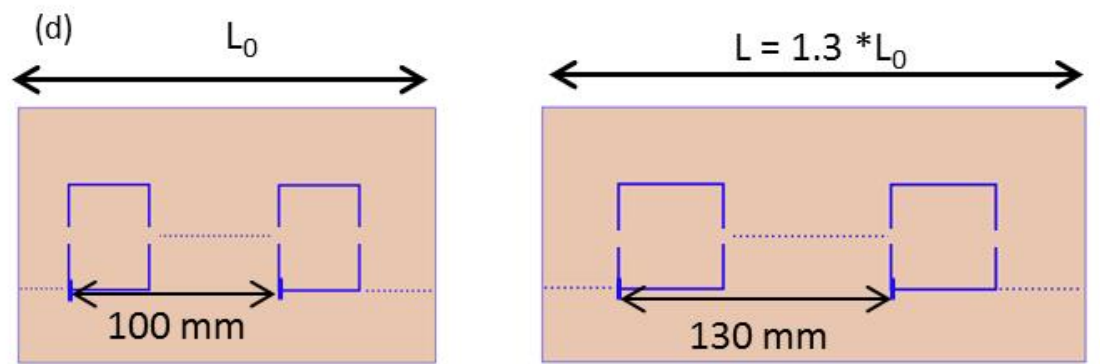
Figure 2 : Numerical simulation used for the evaluation of the curvature modification due to the sensor (a) - Relative error due to the sensor (b)
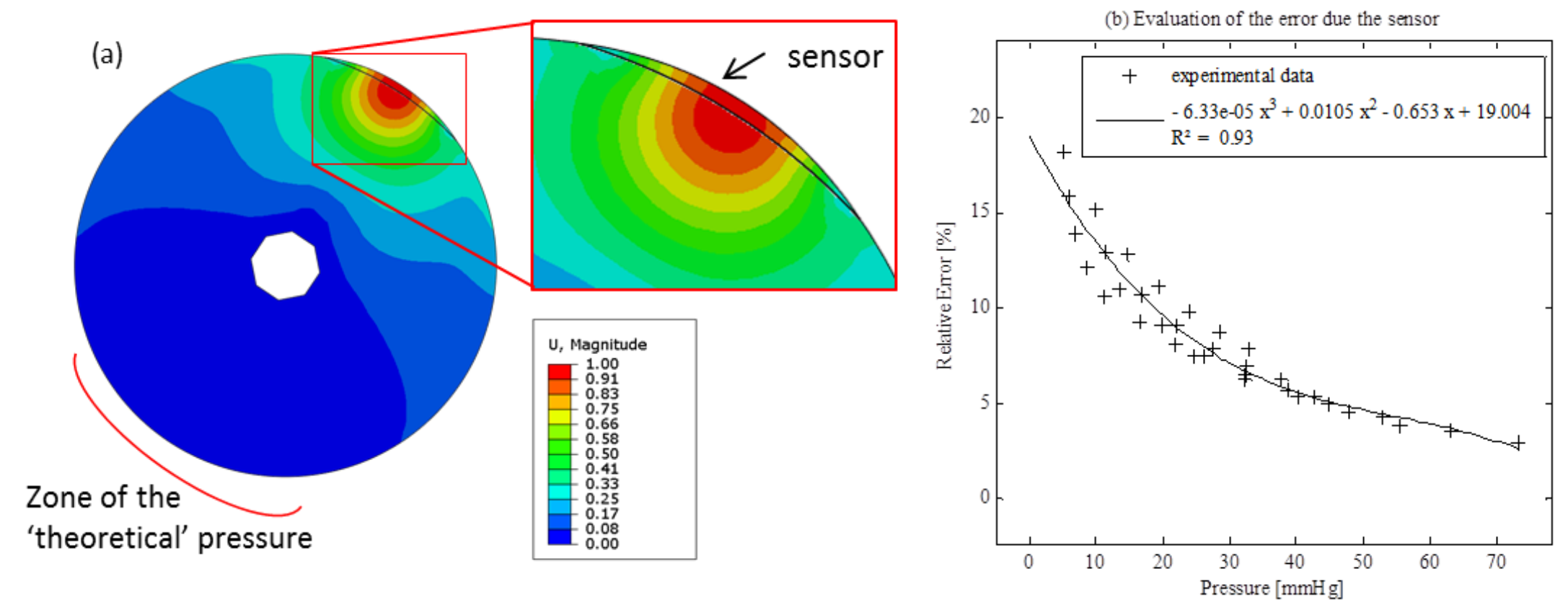
Figure 3 : Building the model geometry: (a) leg given by the 3D scanner - (b) anatomic slice of a leg (* 'Visible Human Server', Computer Science Department, Peripheral Systems Lab., Ecole Polytechnique Fédérale de Lausanne) - (c) bones - (d) leg geometry - (e) bandage geometry

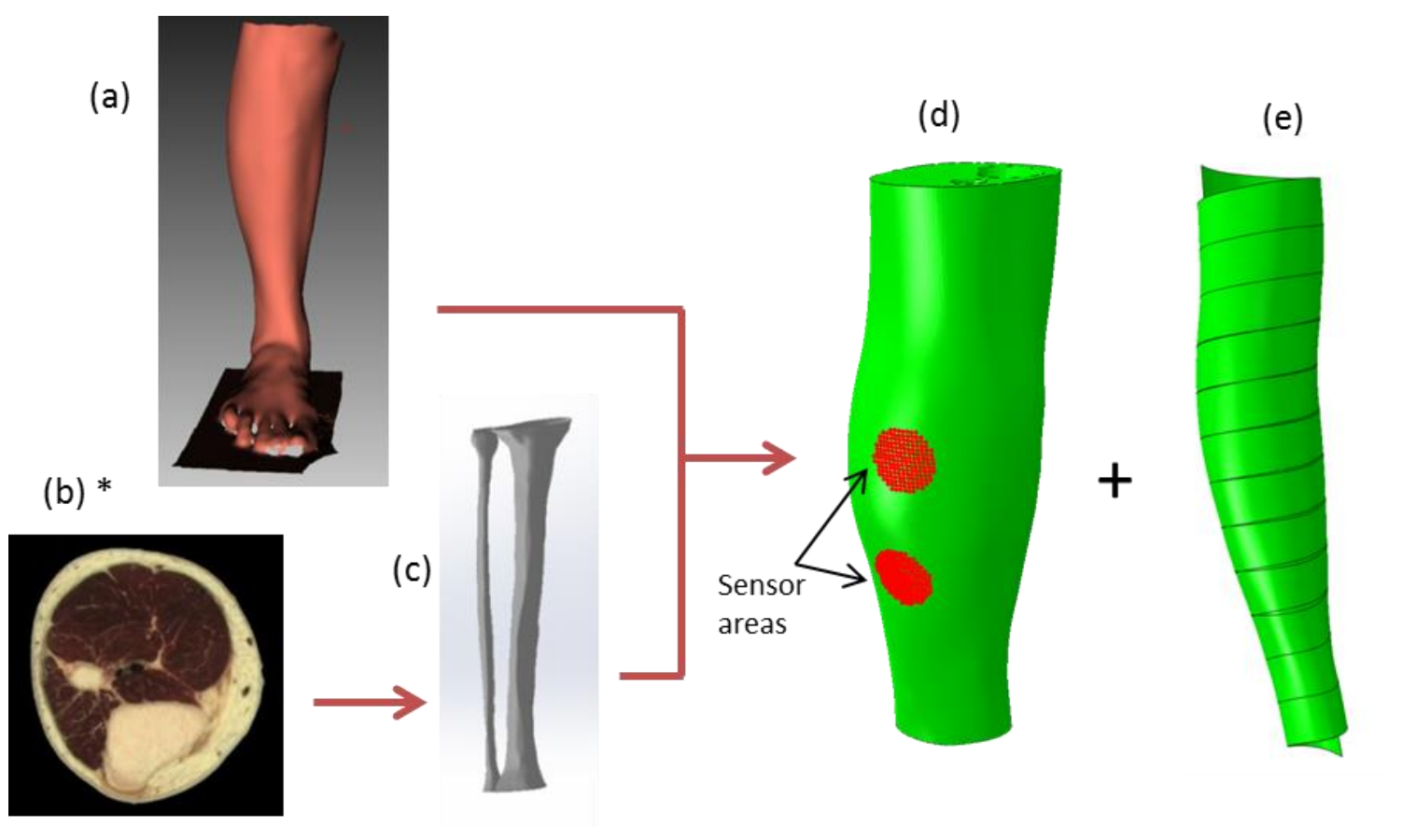


Figure 4 : Different steps of the simulation (a) - (b) - (c) and examples of pressure distribution over the leg (d) - (e)

(a) Bandage dilatation

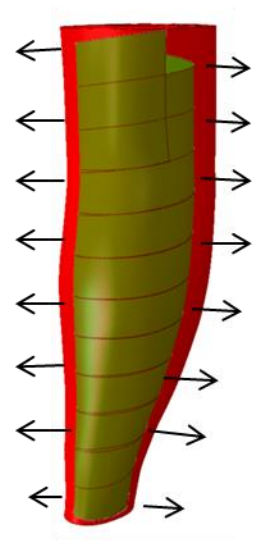

(b) Bandage relaxation and contact activation

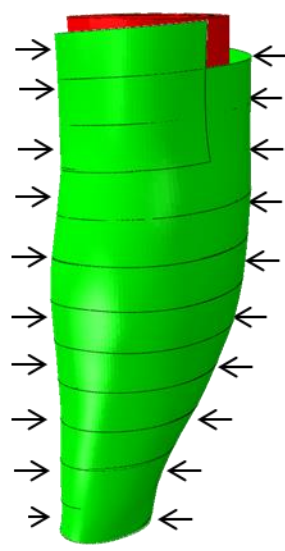

(c) Equilibrium

(d) Biflex 16

2 layers

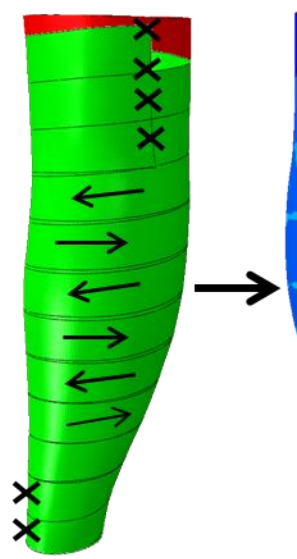

(e) Biflex ${ }^{\circledR} 16$

3 layers

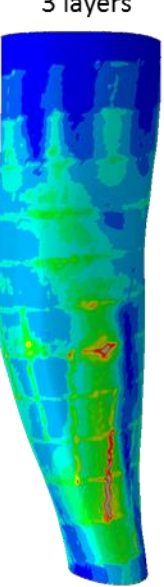


Figure 5 : Experimental results: (a) Mean bandage stretch for both bandages and around both measurement points $B 1$ and $C$, mean bandage stretches for the two different bandages B16 and B17 and mean bandage stretches around measurement points $\mathrm{B} 1$ and $\mathrm{C}-(\mathrm{b})$ Mean pressure values exerted by the four different bandages in supine position and for all measurement points and all subjects - (c) Pressure applied by the B17 bandage vs. the one applied by the B16 bandage - (d) Pressure applied by a 3-layer bandage vs. the one applied by a 2-layer bandage (for the same bandage) - (e) Pressure measured at point $B 1$ vs the one measured at $C$ (for the same bandage and application technique) 
(a) Bandage stretch

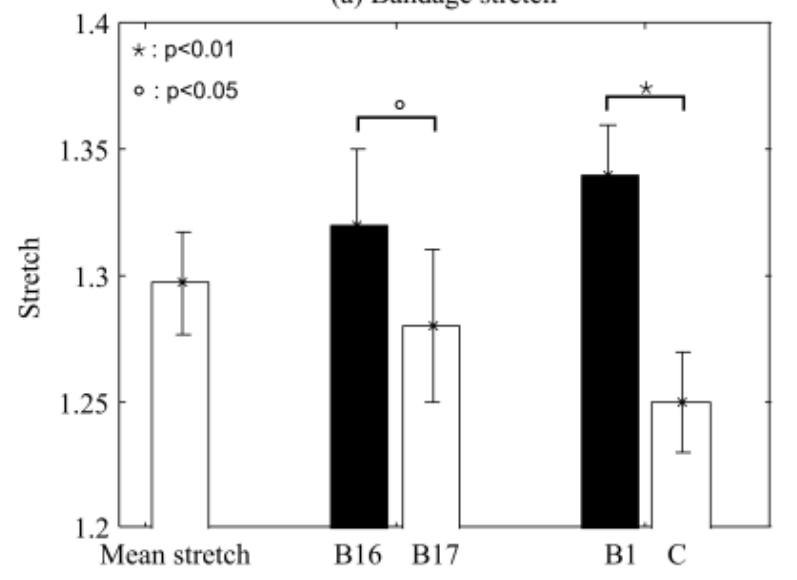

(c) Influence of bandage mechanical properties

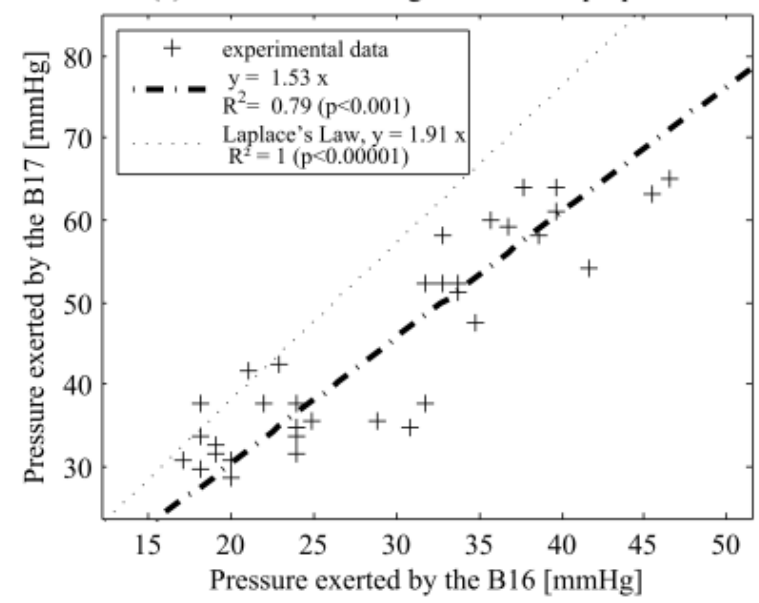

(e) Influence of measurement point

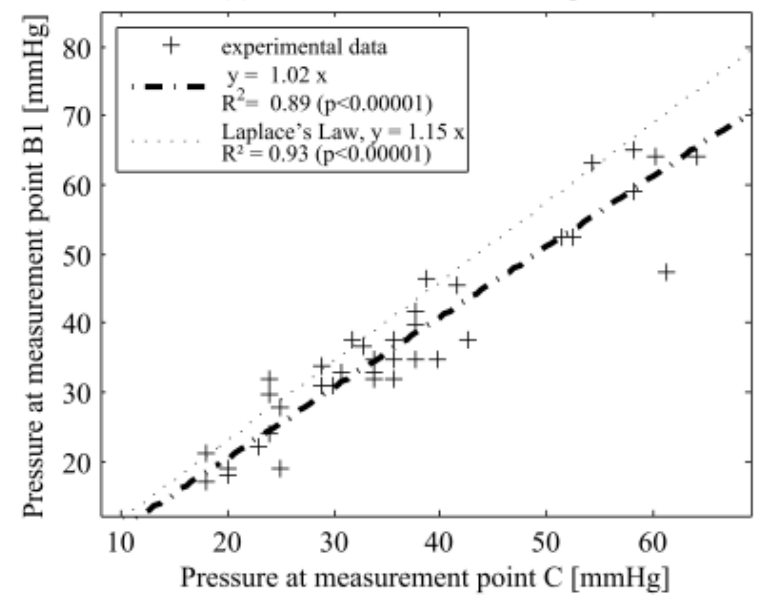

(b) Mean pressures values (all measurement points)

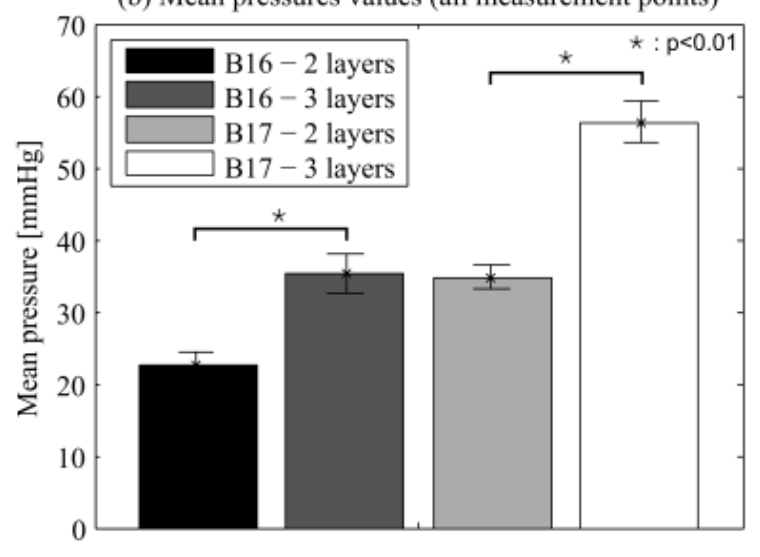

(d) Influence of the number of layers

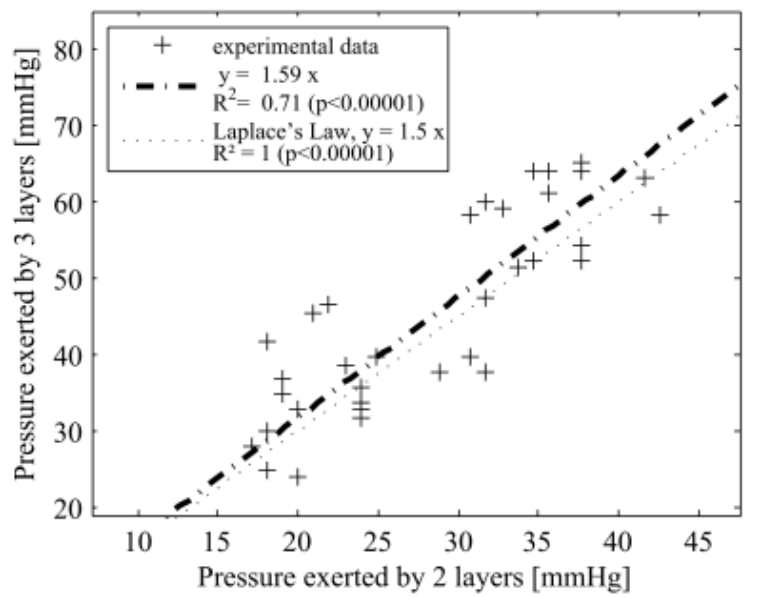


Figure 6 : Results given by the simulation: (a) Mean pressure values exerted by the four different bandages for all measurement points and subjects - (b) Pressure applied by the B17 bandage vs. the one applied by the B16 bandage - (c) Pressure applied by a 3-layer bandage vs. the one applied by a 2-layer bandage (for the same bandage) - (d) Pressure measured at point B1 vs the one measured at $\mathrm{C}$ (for the same bandage and application technique)

(a) Mean pressures values

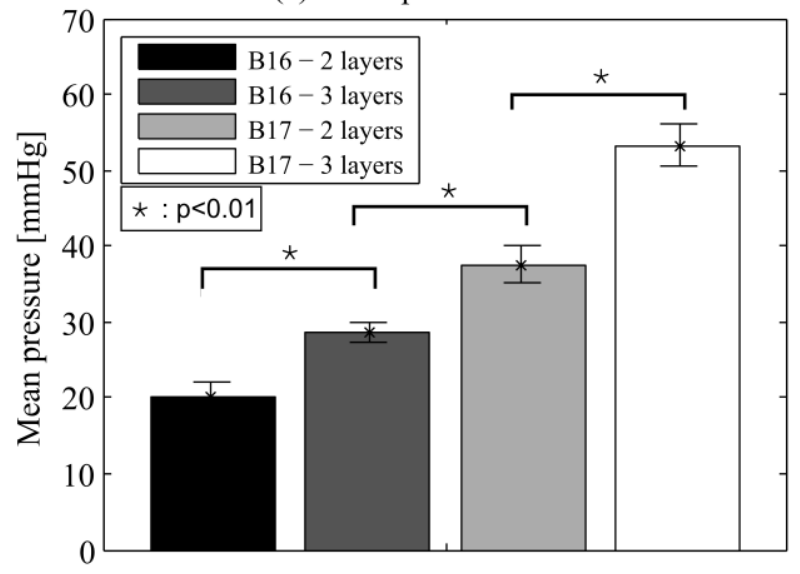

(c) Influence of the number of layers

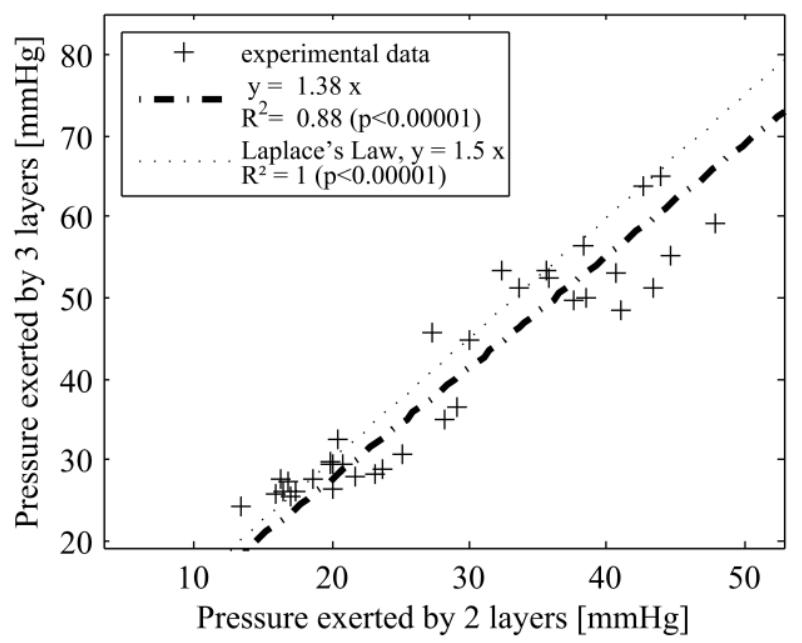

(b) Influence of bandage mechanical properties

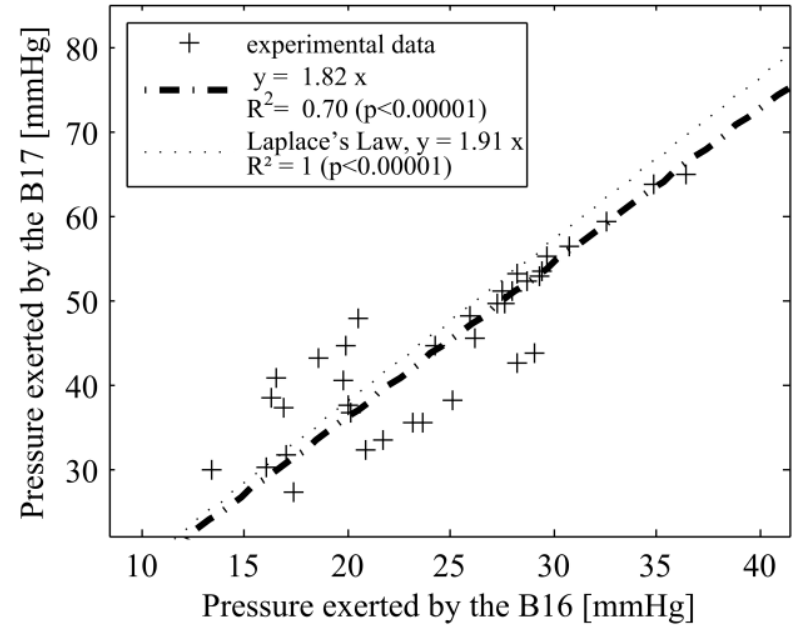

(d) Influence of measurement point

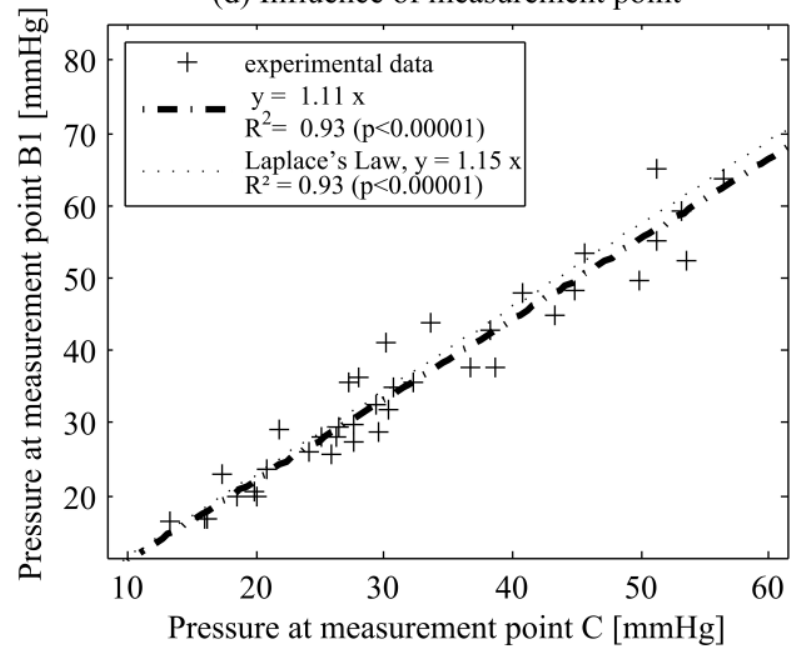


Figure 7 : Comparison of the pressure given by the experiments, the simulation and Laplace's law, at measurement point B1 on the medial side of the leg for the 5 subjects and the 4 bandages

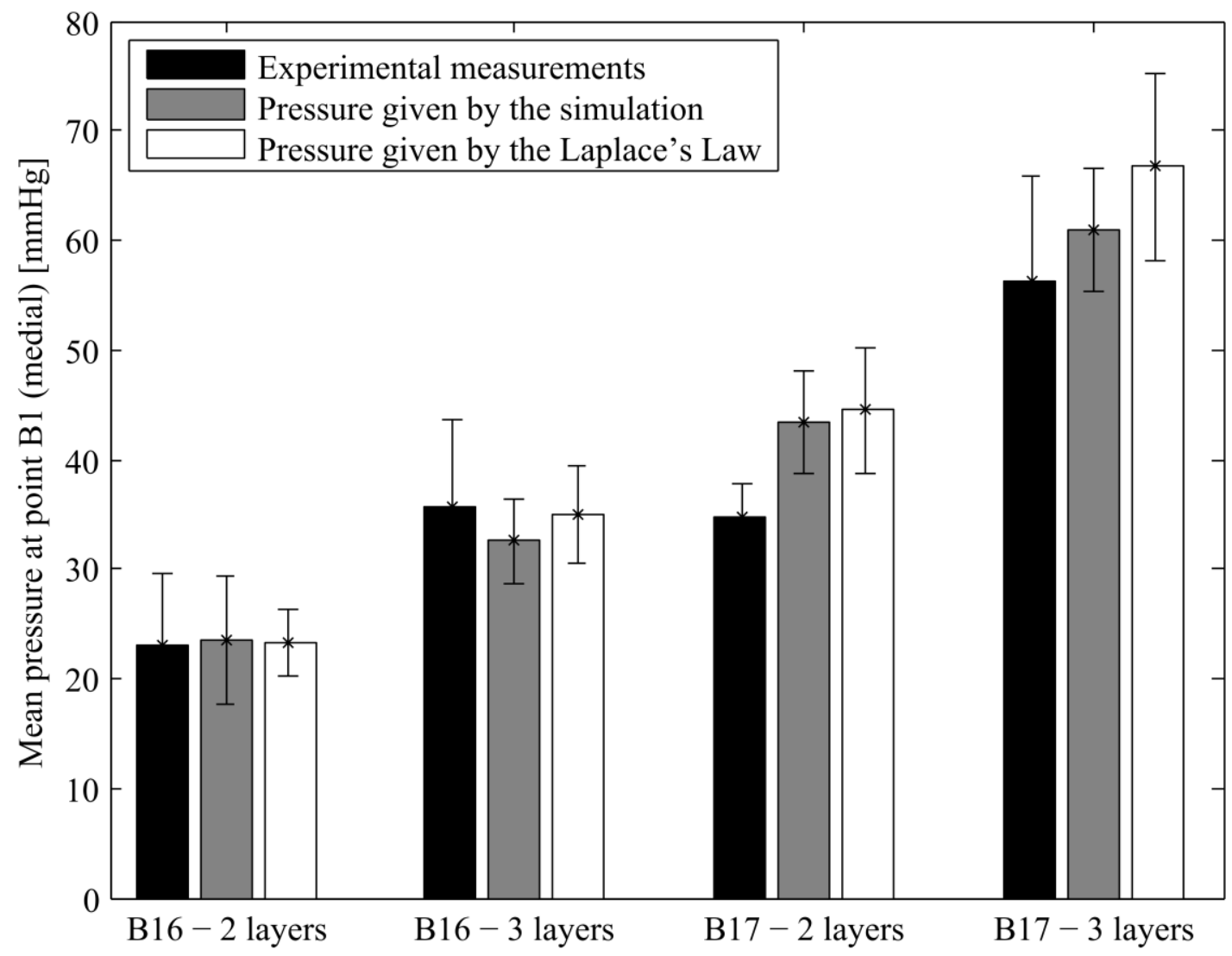


Figure 8 : Coefficients of the linear model for the different data sets ( $\mathrm{n}$ : number of bandage layers, $\mathrm{T}=$ bandage tension and $\mathrm{C}=$ leg curvature)

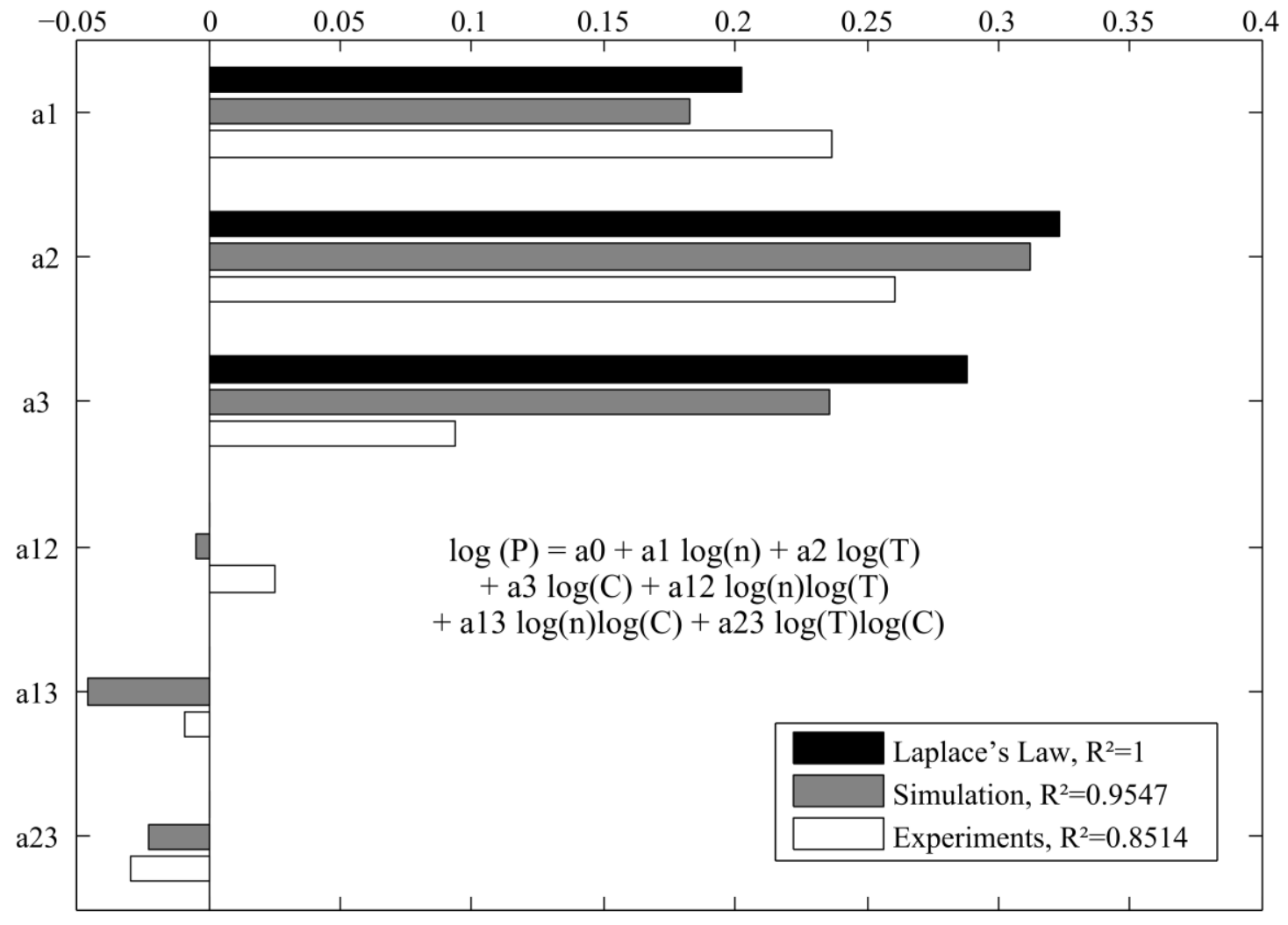

